\title{
Coordination Approaches for CIM
}

\author{
Moira C. Norrie, Martin Wunderli, Robert Montau, Uwe Leonhardt, \\ Werner Schaad, Hans-Jörg Schek \\ Swiss Federal Institute of Technology \\ ETH Zentrum, CH-8092 Zurich, Switzerland, \\ \{norrie,wunderli,schaad,schek\}@inf.ethz.ch, \\ \{montau,leonhard\}@vmeth.ethz.ch
}

\begin{abstract}
We propose a general architecture for Computer Integrated Manufacturing (CIM) based on the coordination, rather than integration, of component systems. The coordination process is achieved through inter-system dependencies controlled by a central, global coordinator. Coordination, like integration, may be either data- or application-oriented. In the case of data-oriented coordination, multidatabase technologies may be exploited to maintain global data consistency. For application-oriented coordination, the global coordinator uses operational dependencies as a basis for the invocation of methods in remote systems. We examine each of these orientations in detail and then provide a comparison of approaches. Specifically, we describe two prototype systems developed in the context of the CIM/Z project.
\end{abstract}

\section{Keywords}

integration methods, coordination model, repository technology

\section{INTRODUCTION}

Traditional approaches to Computer Integrated Manufacturing (CIM) have tended to focus on either a total or a partial integration of component systems. The integration approach may be either data- or application-oriented or some combination of both. In the case of data-oriented integration, all or some of the component systems' data are stored in some form of logical or physical central database. A logical central database is one in which data may be distributed but there is a single, global schema through which the data is accessed. With application-oriented integration, systems are tightly-coupled either by means of direct calls from one application system to another, or, by encapsulating the component systems in a single, global application system.

Generally, the main problems of the integration approach are loss of component system autonomy and a lack of support for system evolution. There are many aspects of system autonomy some of which may be compromised by a particular integration approach. For example, total data integration based on a single global schema is not only expensive in terms of the integration effort but also forces a single enterprise model and this might be considered as a sacrifice of data autonomy. Controlling access to shared data objects by storing them in a central database means 
that the availability of the central database is critical to the continued working of the component systems; this can be described as a loss of operational autonomy.

System evolution can occur through the addition of new component systems or the replacement of existing component systems. To support evolution, it is essential that the general architecture is flexible and that component system dependencies are minimised and isolated. Some forms of integration are too inflexible in that inter-system working is hard-coded and not amenable to change.

In an effort to minimise loss of autonomy and maximise flexibility, we advocate a looser coupling of component systems based on coordination rather than integration. A central, global coordinator responds to actions in one component system by delegating actions to one or more other component systems. For example, the activities of a Computer-Aided Design (CAD) system and a Production Planning System (PPS) system may be coordinated in such a way that the deletion of a CAD assembly results in the coordinator requesting the deletion (or invalidation) of related part lists in the PPS system.

As far as possible, component systems continue to operate as before with coordination performed "behind the scenes". Any changes to the user view of component system functionality arise not from the coordination process itself but rather only in cases where the functionality may be enhanced either in terms of local system extensions or through the introduction of global applications.

The coordination process is based on inter-system dependencies which are stored in the global coordinator's database using a global representation scheme. These dependencies may be viewed, interrogated and updated with relative ease. An important factor is that the global coordinator is not critical to individual system operation. It is the assumption that the coordination activity occurs when significant changes are made to local component system data, e.g. checking-in a design object, or, when global queries are evaluated. Further, we assume that the coordination process is not time-critical.

Just as integration approaches may centre either on data or operations (or some combination of both), coordination approaches may be based on either data dependencies or operational dependencies. In the former case, coordination is achieved through the maintenance of global data consistency. In the latter case, methods in one component system are mapped to methods in one or more other component systems through a global object method server.

In section 2, we discuss the general requirements of CIM in terms of supporting cooperative working and we summarise various approaches to the realisation of CIM systems. Section 3 presents the coordination approach in further detail by introducing a general coordination architecture and an example of the coordination process. Sections 4 and 5 detail two coordination approaches which have been investigated in the context of the CIM/Z project (Integration of Databases and CIM Component Systems) at ETH Zurich (Schaad, Montau, Wunderli, Leonhardt \& Lüthi 1993). The first of these is an approach based on data dependencies and the exploitation of multidatabase technologies and is described in section 4. The second approach, based on operational dependencies, is described in section 5. Section 6 provides some general comments on the two approaches. Concluding remarks are given in section 7 .

\section{CIM REQUIREMENTS AND APPROACHES}

The manufacturing process spans many departments and activities of an enterprise. The department structure reflects the decomposition and distribution of activities and, correspondingly, separate application systems have been developed to support the activities of these departments. Enterprise structure is not the only reason for the evolution of independent application systems. Manufacturing systems cover a wide range of activities, both technical and management, and these may have very different requirements in terms of the amount and type of data, the functionality, the run-time environment and performance. Consider the two main tracks of information flow shown in figure 1 . 
The economic and administrative track comprises data which is usually organised within complex but monolithic applications purpose-built for the tracking of the complete economic life cycle of a product. Because of the integrated nature of these so called Production Planning Systems (PPS) (or Management and Resource Planning Systems (MRP)), data exchange and data consistency between the different production phases has not been a problem. Even current efforts to decompose and modularise these applications does not effect the data consistency and the capability to exchange data between the modules. So we can regard data exchange and data consistency as guaranteed on this track of information flow.

On the technical track, we find a quite different and much more complex situation. Here, the applications are highly specialised. The primary purpose of a component system is not to exchange or synchronise data but rather to fulfil, in an efficient way, a specific application task, such as the design activity (CAD) or the programming of numerically controlled machines for a CAM process. Often data exchange consists of physically transferring files on disks between departments or data may even have to be reentered because of the heterogeneity of the computing environment.

A slightly different view of the information flows in the production process is taken by Scheer (Scheer 1990). Scheer divides the production process of a product into two phases, the planning phase and the manufacturing phase which is reflected in both the economic and the technical track. Figure 2 shows in more detail

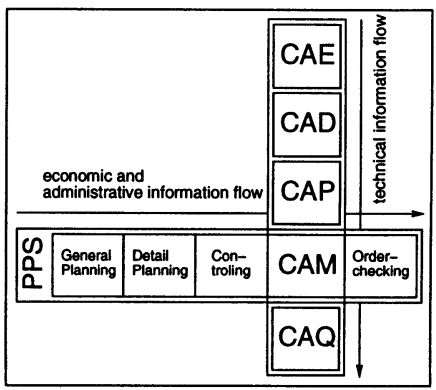

Figure 1: Data sources in manufacturing the required tasks (and departments) of the manufacturing process and the supporting component systems.

It has been found that the decisions taken in the planning phase, although directly causing only about $10 \%$ of the total production costs, have a high indirect effect on the total cost of a product because of their influence on the manufacturing phase. At the start of the manufacturing phase, all decisions for the ergonomics, functionality and production costs of a product have already been taken, either directly or indirectly. So the main purpose of this phase is to fulfil the directives defined by the planning phase.

Increasing national and international competition has led to the shortening of product life cycles and an increase in both product complexity and the importance of cost efficiency

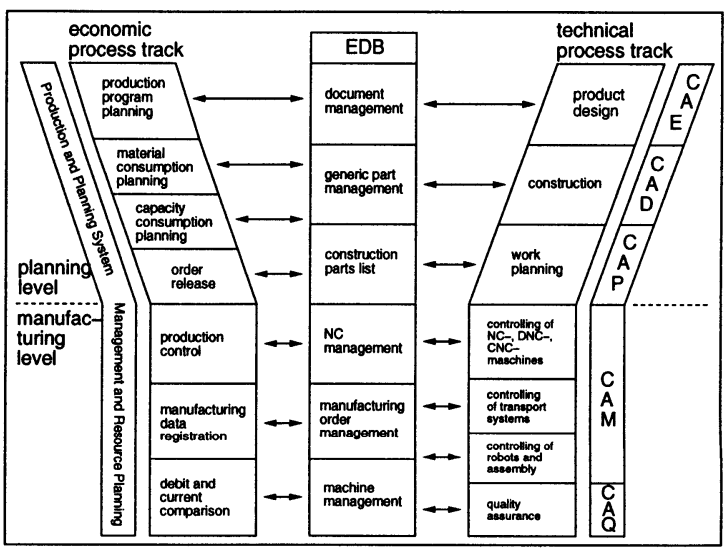

Figure 2: Data sources in manufacturing and flexibility in production. As a result, the necessity of standardised product data exchange and automatic data consistency control has become a prerequisite of a successful enterprise.

The problem of product data exchange between CAx component systems has been tackled by different national and international standards. As examples, we mention the 'International Graphics Exchange Standard' (IGE 1988), the 'Standard d'Échange et de Transfert' (SET 1984, SET 1985) and country specific subsets like the 'Verband 
Deutscher Automobilindustrie - IGES Subset' (VDA 1987a) and the 'VDA Flächenschnittstelle' (VDA 1987b); these are currently used for the exchange of data but are more or less restricted to 2D/3D geometric data. However, the upcoming ISO Standard 10303 STEP/EXPRESS (STE 1992), which covers the exchange of product model data in total, is intended to replace all the currently used product data exchange standards and so to solve the problem of different, partly overlapping, partly disjoint formats. Note that these standards are not meant to ensure data consistency across CAx systems' data. Nevertheless, they can serve as the foundation for tools built for that purpose.

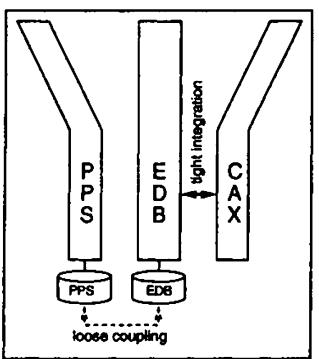

Figure 3: Current EDBs

In the classic CIM strategy, data could be integrated into one central database. However, in practice, such a total integration is not achieved mainly because of variation in requirements, complexity and the self-evident need for the use of existing systems. Additionally, PPSs are very large and well-established and therefore must be retained. For these reasons, the economic and administrative data is kept separate from the technical data. This is commonly referred to as the Engineering Database (EDB) approach and is illustrated in figure 3 .

The problems with this approach are twofold. First, the EDB is critical to the operation of all technical component systems. Any failure occurring in the EDB means that work on component CAx systems cannot proceed. Second, usually there is little or no coordination between the PPS and the EDB and therefore global consistency of the CIM system is left to management control. Further, as discussed before, the integration of all the technical data into one system may result in a certain degree of inflexibility in terms of replacing component systems with alternative products.

The recommendation is to leave component systems with their local data and only integrate some of it into the EDB as illustrated in figure 4 . To avoid an unnecessary replication of data, the EDB may consist primarily of references to local data objects and any data required to support extensions to the functionality of component systems. An example of such a functionality extension would be to allow users of a CAD system to query part information from other component systems to locate parts with designs which can be modified to give new designs. Due to the fact that only $25 \%$ of a design is new in terms of functionality, reusability can be applied in at least $75 \%$ of the design task.

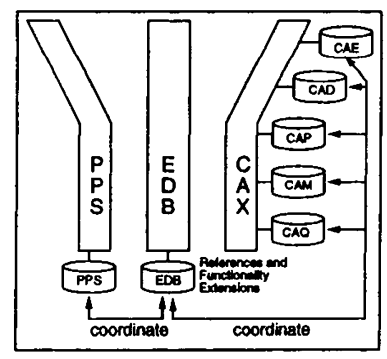

Figure 4: Recommended data handling for EDBs

For global consistency in a CIM system based on this approach, coordination is required between the technical CAx systems and the EDB and also between the EDB and the PPS. To achieve this, we propose a coordination architecture which minimises the degree of integration thereby increasing the autonomy of component systems and flexibility in terms of the introduction and replacement of component systems. This approach is based on inter-system dependencies which are stored in a central coordinator in a global representation language. Dependencies among systems can easily be queried and updated. We describe the coordination approach in further detail in the following section. In the context of the CIM/Z project, we have investigated two basic coordination approaches: one is data-oriented in that it is based on data dependencies and the other is method-oriented in that it is based on operational dependencies. Prototype systems have been developed for each of these two approaches and these are described in sections 4 and 5 respectively.

Other related projects include the following. The concept of using an integration database was used in a project at IBM Germany, at the Heidelberg Scientific Centre (Brosda 1992, Brosda \& Herbst 1992). The use of enhanced database support (e.g. by using complex objects) for CIM was invest- 
igated at IBM Almaden Research Centre, San Jose (Lorie \& Bever 1987). The integration of CAD and Production Planning Systems through the use of update dependencies was studied in a project at the University of Maryland (Mark, Roussopoulos \& Cochrane 1994). Achieving coordination through the use of a central, integrated document and order management system was investigated in the DOCMAN project at the Technical University of Aachen (Eversheim \& Grosse-Wienker 1991).

Our data-oriented approach relates to ActMan (Jablonski, Ruf \& Wedekind 1988, Jablonski, Ruf \& Wedekind 1990), a project at the University of Erlangen-Nürnberg, Germany. This project investigated a form of bilateral coordination between different CIM component systems. An important goal of our project is that we want to increase the local autonomy for the component systems, whereas, in ActMan, a tight coupling between the component systems was chosen. For example, ActMan uses a central, global database where globally important data is replicated. We avoid this approach and keep, whenever possible, all the data in the data management system of the component system. We then increase the local autonomy by using an advanced transaction model rather than the two-phase commit protocol as used, for example, in ActMan. Further differences stem from the fact that both projects use different concepts for global constraint management.

\section{COORDINATION ARCHITECTURE}

In this section, we outline the general architecture of a coordination system and the functionality of the two main kinds of component through which coordination is achieved; these are the CIM Agents and the Global Coordinator. Figure 5 shows the general coordination architecture; for simplification we show only two component systems.

As an example of coordination, we consider a CAD system and a PPS. The CAD system supports the design activity and stores information about the various $\mathrm{CAD}$ drawings consisting of $\mathrm{CAD}$ assemblies and their components. One of the PPS' tasks is to manage information about the structure of parts. There is a dependency between the two systems; part data referred to in the CAD drawings must exist in the PPS. If a part is discontinued, then we must

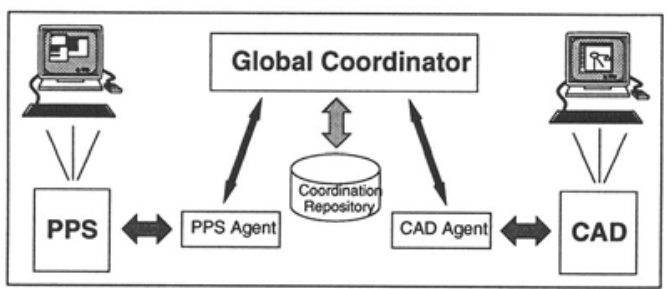

Figure 5: General Coordination Architecture ensure that designers cannot reference this part in future designs - and we must somehow inform designers that existing designs using this part are no longer valid. This is an example of an inter-system dependency which is the basis of the coordination process and will be stored in the Coordination Repository.

The main task of the Global Coordinator is to ensure, with the help of the CIM Agents, the consistent state of the CIM system. The Global Coordinator does this by coordinating activities of the various component systems according to various inter-system dependencies. Each component system has a CIM Agent which monitors local activity and notifies the Global Coordinator of any actions that are pertinent to the inter-system dependencies. Based on these dependencies, the Global Coordinator will delegate necessary actions to one or more component systems via their CIM Agents.

A CIM Agent provides the coordination interface for a component system. As illustrated in figure 6, a CIM Agent must be able to communicate with its component system and also with the Global Coordinator. One of the major functions of a CIM Agent is to map between local representations of operations and data to the global representation scheme. Local system dependency is therefore isolated in part of the local CIM Agent and the rest of the coordination components are general and not specific to particular component systems. 
A CIM Agent may have its own repository to record information about which local operations and data are relevant to coordination, logs of local activities and any other information required to perform its part of the coordination task.

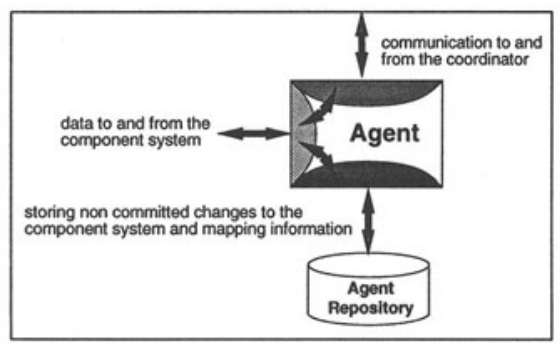

Figure 6: General Architecture of Agent
Global transaction management is a prerequisite to ensure that all information about changes and the corresponding coordination activities reach the relevant parties and are acted upon. Since a component system may not be a database application system and therefore may be without transaction and recovery support, it may be necessary that its CIM Agent takes over the role of providing some form of logging and atomicity control. The issue of global transaction management is an important part of the $C I M / Z$ project and has been investigated in the context of the data-oriented coordination approach which is discussed in section 4 .

The coordination architecture presented in this section is very general and does not specify either the form of inter-system dependencies or the methods of communication between the various component systems, their CIM Agents and the Global Coordinator. We now go on to discuss two specific architectures - one based on data dependencies and one based on operational dependencies.

\section{COORDINATION THROUGH DATA DEPENDENCIES}

In the data-oriented coordination approach, the activities of the CIM component systems are coordinated through the maintenance of system-wide data consistency. Here we describe a particular data-oriented coordination prototype, CIM/DB, that has been implemented as part of the CIM/Z project (Norrie, Schaad, Schek \& Wunderli 1994a, Norrie, Schaad, Schek \& Wunderli 1994b). CIM/DB adopts a "database approach" to the CIM problem and exploits multidatabase technologies. To illustrate the approach, we return to the example of the CAD and the PPS systems discussed in the previous section. There is a data dependency between the two systems; parts referred to in the design assemblies of the CAD system must exist in the PPS. Such a dependency is expressed as a global data constraint and the Global Coordinator must be informed of any updates within a component system that may result in a violation of such a constraint.

The Global Coordinator stores in a coordination repository information about the various component systems, their schemas, the global constraints and reactions on constraint violations. In addition, it is necessary for the coordinator to store information about the individual relationships between data objects of the component systems. For example, it must record the relationship between a particular part list of the PPS and a particular design of the CAD system. When the Global Coordinator is informed of local component changes, it delegates actions to be taken by the appropriate CIM Agents in order that consistency be maintained.

The Coordination View is a conceptual model of the Global Coordinator's data. It is expressed in terms of the semantic data modelling language NIAM/RIDL (Wintraecken 1989). Figure 7 shows the part of the Coordination View that describes object types and the constraints in which they are involved. The solid circles represent entities while boxes represent relationships between those entities. The dashed circles represent attributes. For example, each ISA constraint is represented by an ISA_Constraint entity which has an attribute ISA_Constraint_Type that indicates the category of ISA constraint (disjoint, total etc.). Bars above relationship boxes are used to specify relationship cardinalities. For example, the placement of a single bar above the relationship between ISA_Constraint and ISA_Constraint_Type indicates that the relationship is one-to-many, i.e. an ISA constraint has a type, but there 
may be many ISA constraints of that type. The ' $\mathrm{V}$ ' on the ISA_Constraint edge indicates that every ISA constraint must have such an attribute.

Each global constraint is represented by a constraint entity and is classified as either a relationship constraint (Relationship_Constraint) or a subtyping constraint (ISA_Constraint).

An example of a relationship constraint is shown in figure 8: A CAD assembly must have at least one, and possibly many, associated part lists while a part list is associated with exactly one CAD assembly.

In addition to the representation of global constraints, the coordinator repository must record the actions to be taken on the violations of such constraints. For example, it might associate with the constraint of fig-

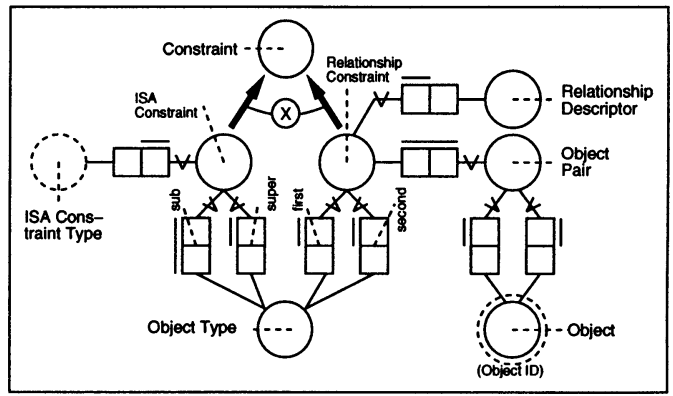

Figure 7: Part of the Coordination View ure 8 that a violation of this constraint resulting from the deletion of a particular CAD assembly should invoke actions to delete all of the associated part lists. Then the coordinator would send messages to the appropriate CIM Agents requesting them to initiate local operations to delete the relevant part list objects. This would all be performed within a global transaction to ensure that either all of the local deletions are performed, or, the original deletion of the CAD assembly would be undone and the user initiating the CAD delete operation would be informed of the failure of the delete and some compensating local action would be performed under the auspices of the local CIM Agent.

The inter-system data dependencies can be thought of as having both static and dynamic parts. The static part is expressed as global constraints that should be satisfied by the system. The dynamic part specifies actions to be taken by the Global Coordinator to restore global consistency in the event that an action in one component system causes one or more constraints to be violated. Further details of the data dependencies and the language used to define them is given in (Norrie \& Wunderli 1994).

The actions known to the Global Coordinator are high-level logical operations which are mapped by the CIM Agents to one or more local operations. For example, the Global Coordinator may request the deletion of an object in a component system. It is possible that this is mapped

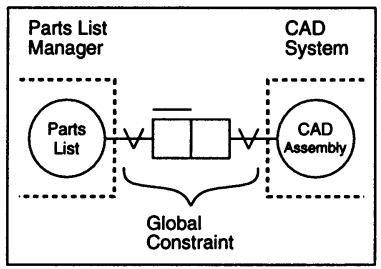

Figure 8: Relationship Constraint

directly to a database delete operation on the local object. However, it is also possible that, in a specific component system, it is mapped to a sequence of local operations that involve manual intervention such as requesting a local system administrator to perform the delete and notifying local users.

The actions to be taken by the Global Coordinator in the event of a global constraint violation are specified in the Global Coordinator's repository. It would be very inconvenient if every reaction description for every combination of constraint and violating action had to be specified individually. For this reason, default reactions for the various forms of constraint and violating actions are predefined so that they ensure a consistent database state in terms of the global data model.

It is the responsibility of a CIM Agent to notify the Global Coordinator of updates on objects relevant to the global data constraints. This communication requires an exchange model and language, such as STEP/EXPRESS (STE 1992), for communication between the coordinator and agents - but we shall not detail this here. The CIM 
Agent has a local NIAM/RIDL schema which describes those parts of the local data set relevant to system-wide consistency; this defines the Coordination Interface of the component system in terms of globally important objects.

The interface between a CIM Agent and its component system is less flexible than those between a CIM Agent and its repository and between the agent and the coordinator since it involves interfacing to existing systems. To understand how such an interface can be realised, we outline one of the component systems of CIM/DB - the CAD system Pro/Engineer (Par 1993). The objects managed by this CAD system are, for example, assemblies, parts and drawings. Initially, a user either calls a search/retrieve operation to load an existing object or they create a new object. An object in memory can be modified by calling a sequence of predefined update operations. There are also operations to query an object and to create reports about its components. On completion of their task, the user can call a store operation to save the changes to the $\mathrm{CAD}$ object.

To perform the coordination task, a CIM Agent has to take over the role of a "spy". It has to monitor the operations that are executed and, based on its model of globally important objects, must recognise operations of global interest. For example, for the part lists objects stored in the PPS, it is important to know when a part or subassembly is added to or deleted from an assembly in the CAD system. Then the CIM Agent must be aware of calls to operations to create, assemble, disassemble or delete parts and subassemblies.

In the case of Pro/Engineer, the CIM/DB component system is enhanced with procedures that are triggered whenever the associated operation is executed. Such a notification procedure can be installed so that it is called either before or after the execution of the associated operation; it sends a notification message to the CAD Agent with the name and the parameters of the operation. The agent receives the message, records it in its repository and evaluates the message. It specifies the operation and its parameters in terms of the global data model ready for communication to the Global Coordinator.

The CIM Agent also has to respond to actions delegated by the global coordinator. The operations and objects of the coordinator's request must be mapped into the language of the component system. For example, consider the case where the coordinator sends a message to the agent of the CAD system to remove a part from an assembly. The agent searches for the local names of that part and assembly in its repository. It calls a read operation for this assembly to load it into the memory and then a disassemble function is called to remove the part from the assembly. Finally, the modified assembly is stored. Information is saved in the repository in order that the user can be informed of the changes when the assembly is next accessed.

The issue of transaction management is how to ensure the atomicity and durability of global transactions, specifically in the case where one or more component systems do not support transaction management. A global transaction arises when a CIM Agent informs the coordinator of an operation on a globally important object. Some part of the coordination activity may not complete successfully either because of failure or due to the coordinator or some component system denying a requested action. Atomic transaction execution is the only guarantee that a change performed in one component system is propagated to the other component systems. The durability of global transactions ensures that once such a coordination activity has been completed successfully, its effects will not be lost.

If a component system is using a DBMS, the same DBMS may be used to store the agent's repository. A modification performed on both objects in the component system and objects in the repository can be performed inside a transaction thereby ensuring local atomicity. Additionally, the use of the component system's DBMS for the agent's repository avoids the replication of data. In the case where all component systems use a DBMS, the problem of building transaction management on top of existing DBMSs corresponds to the multidatabase problem (Breitbart, Garcia-Molina \& Silberschatz 1992).

Component systems without any DBMS normally also do not support transactions. Some of these systems write all operations and their parameters to trail files. If the system crashes, these files are read after the restart and all operations are re-executed. This helps to avoid the loss of data locally but does not guarantee consistency if the component system has been involved in coordination activities. We therefore consider a slightly extended multidatabase problem where not all component systems support transactions. 
If a component system has no transaction support, its CIM Agent monitors the system and logs local operations in its repository which may be considered stable as it is managed by a DBMS. In the case of a local abort, the agent checks the log after restart. If there is an entry about the start of an operation, but no entry about the completion of this operation, then the abort must have happened during the execution of the operation. It is possible that a global transaction had already been started in which case a begin-of-transaction would have been written to the log. The agent, together with the coordinator, can determine the state of the global transaction and, using a global commit protocol, terminate the global transaction. If the global transaction was completed successfully, the agent has to ensure that the local operation also completes. This is done by checking the version of the relevant globally important objects and, if necessary, re-executing some parts of the local operation. If the global transaction was aborted, the agent has to undo any local changes by reinstating the old version or, as an alternative, restarting the global transaction and completing the operation. We have built a CIM Agent for Pro/Engineer based on this approach.

The second aspect of transaction management is to isolate transactions executing in parallel. Rather than using strict two-phase locking (2PL) together with two-phase commit (2PC), which would severely restrict the local autonomy, we use open-nested transactions (Weikum \& Schek 1992). Open nested-transactions have the advantage that subtransactions can be committed early, thereby allowing other transactions to access the objects in the database. Because, in some cases, committed subtransactions have to be compensated if the global transaction aborts, the system has to ensure that compensation will be possible. This is done by using multi-level transaction management (Weikum 1991, Beeri, Bernstein \& Goodman 1989). By exploiting the semantics of the transactions, the global transaction manager decides what subtransactions are compatible and can be executed together. We have extended this idea so that we can have a mixed execution of global and local transactions (Schaad \& Schek 1993, Schek, Weikum \& Schaad 1991). This is necessary if we want to use this concept for existing component systems.

\section{COORDINATION THROUGH OPERATIONAL DEPENDENCIES}

The use of data dependencies, as described in the previous section, is only one way to obtain consistency in a CIM environment. Alternatively, one can focus on operational dependencies to ensure consistency by coordinating at the level of application methods. For this, it is necessary to know how data is accessed by the operations in a component system.

In order to discuss this operational approach of coordinating CIM component systems, the idea of global objects and methods must be reaffirmed. An object is of global interest if it is important for more than one component system. Therefore, access to global objects requires system-overlapping actions. For example, the insertion of a global object in the CAD system may require another insert in the PPS.

Any access to objects requires a method and these methods are usually kept in the method bench of an application. A method is defined as a self-contained function which performs an operation belonging to an object. These object methods include every operation to create, delete or manipulate an object (for example creating a point or a line) (Shooman 1983). In the case of relational database systems, an object is a tuple of a relation and an associated method could be a simple insert procedure. For complex objects which affect more than one relation, the corresponding method will also be more complicated involving access to several relations.

To achieve the various aims of connecting component systems in the CIM environment, a number of requirements have to be fulfilled. Primarily, a component system must be able to distinguish local and global objects and their methods. In the case of accessing a global object method, an appropriate action in the coordinator has to be initiated by sending the relevant information on the object, the required object method, their parameters and the initiator to the Global Coordinator. The coordinator then has to determine and initiate the requisite local methods in all of the involved component systems. During the execution of such a global object method, the task of the Global 
Coordinator is to ensure the ACID-properties (atomicity, concurrency, isolation, duration) of transactions in order to perform global commits or aborts.

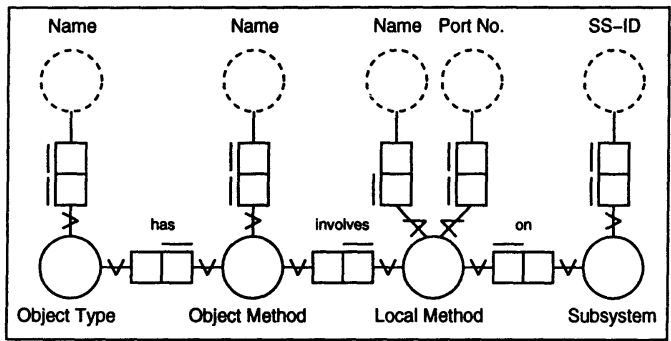

Figure 9: Metamodel for method server
To enable calls of methods between different systems, the Global Coordinator requires knowledge about the coherence between the systems, the relationship between global methods and local methods and the functionality of managing the control flow between the systems. In addition, the Global Coordinator must be able to find out where the required local methods reside in order to access them. Therefore, the Global Coordinator must store information about the global object methods associated with a global object and the involved local methods with their location, which is mapped to a port number, and the identifier of the component system to which they belong. The attachment of actions to component systems should be done in such a way that it is easy to view and change them and hence support system evolution. For that reason, the operational dependencies are described through the relationship of global and local methods as shown in the simplified NIAM-model for the Global Coordinator of figure 9.

Through this model, maximum flexibility of connection can be attained. If the Global Coordinator receives a request for a global object method, it only has to look for the required local object methods and initiate them. Further, the addition or deletion of a component system only requires a simple update to the Global Coordinator's database once the required local method has been implemented. For example, assuming a relational database for the Global Coordinator, only a single tuple has to be changed. In the case of migrating a component system from one computer system to another, only the location addresses (Port No.) of the local methods have to be updated. However, we note that, while it is very simple to realise a Global Coordinator in this way, it disregards possible transaction and communication problems.

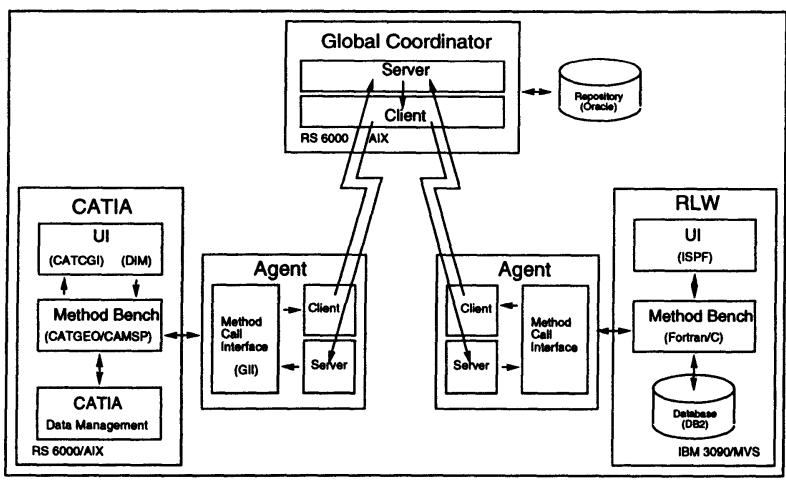

Figure 10: General architecture for a method server
To enable a system-overlapping access of methods, a reliable mechanism for the heterogeneous environment of current CIM systems is required. The standardised Remote Procedure Calls (RPC) (Bloomer 1992) can be used; these allow a method call in a remote computer system with different hardware and operating system from the source system. The RPCs are based on the TCP/IP network protocol, a common standard for data transfer in a heterogeneous computer environment. Almost every system provides an interface for net- 
work communication based on TCP/IP and the IP protocol is still the only way to obtain a really dynamic routing in a network (Gorys \& Brauch 1993) which is a prerequisite for this coordination approach.

RPC use the client-server principle and the general architecture of coordination given in section 3 is elaborated in figure 10. The architecture indicates how RPC can be embedded in the coordination concept for an example of two component systems which will be discussed later.

A component system must know the network address of the Global Coordinator which must have an independent server process waiting for any calls to global object methods. The Global Coordinator must be able to call all the associated local methods, even if they are located on different hosts. Every CIM Agent must have a server process running which is prepared for any call of the local methods. These server processes can be accessed from the Global Coordinator by their internet addresses (SS-ID) and their port numbers.

In addition to the remote call of methods, the data part, which is the method parameters, has to be transferred. In a heterogeneous environment, there may be different ways of representing the standard data types like integer, character, etc. For this, the XDR standard from SUN-Microsystems is useful; it defines routines to translate between the standardised external representation and C-Variable-types which may be stored in different system-dependent codes such as ASCII or EBCDIC.

Another problem is the difference in the parameters of the local methods from those of the global method. A global valid format for the parameters of a global method is required. This global format for parameters consists of the particular parameters, their data type and their position in the transferred data stream. To start a global method, the client of the initiating agent has to map the format of the parameters from the local format to the global one. This global format can be described as the global language which the coordinator and every involved component system of a global method must understand. In the event of a global request arriving at a CIM Agent, the server part has to map the incoming data stream to the format required by the associated local method.

To illustrate how this coordination approach based on operational dependencies can be used, we now consider two examples. The first one deals with the maintenance of global integrity constraints and the second one gives an example of how the functionality of a component system can be extended. The component systems are the CAD system CATIA running on an RS/6000 environment and an EDB-like prototype, RLW, developed at the CADETHcentre of ETH Zurich. RLW supports the management of part data, including structural data for part lists as well as classification data and the management of documents.

For the first example, consider the insertion of a new part in a CAD model during a CATIA session and the following release of the technical drawing. In the case of adding a new part to the CAD model of a group drawing, the part data inserted must also be inserted in the part data management of the RLW-system. To achieve a mechanism for consistency over geometrical data in $\mathrm{CAD}$ and non-geometrical data stored in a relational database, the functionality of CATIA had to be extended. All of the existing geometrical elements can be grouped together persistently to the user-defined data type or feature "part" through an additional CATIA-function using the GII (Graphical Interactive Interface) application interface of CATIA (GII 1988, CAT 1992). For such a defined part, the relative data, such as name, part number, material and manufacturing restrictions, and the structural data for the part list can be inserted.

Since the relative method is a global one, the action has to be sent as a request for a global method (insert part) to the coordinator. The coordinator determines that for this global method, the local methods of RLW to insert part data and insert the associated structural data are both required and initiates them accordingly.

After this action, the user might want to finish his work on the CAD model and release it. Again this operation is of global interest and, after notification, the coordinator activates local methods of RLW in the modules for part and document management to update the status of all related data. Naturally, in the CAD system, the access possibilities of the model also have to be changed in order to ensure the consistency of the component systems cannot be violated by future changes.

In contrast, the second example focuses on the extension of component system functionality. Consider the case of searching for a similar part during a CAD session. The user has to describe the characteristic features of the 
required part. This he does using shape properties in the case of a single part or using functional properties in the case of a group part. This search request has to be sent through the Global Coordinator to RLW where the relative parts can be determined in the classification module. The result of this query is the set of part identifications and some characteristic features and these are returned to the CAD user. Note that $R L W$ supports a part number mapping which maps the internal identification to an external part number system which conveys more information to the user (Montau 1993, Montau 1994). After accepting a part in CATIA, the global method to find out the identification of the CAD model related to that part occurs and this initiates a query in the document management of RLW. In the case of success, the model identification is given back to the CATIA-application and the geometry of the part can be shown. If the user is satisfied with the result, he obtains a copy of all the CAD data in a so-called detail workspace of his current model. In this way, the costs and the time to market can be reduced as there is no further need to design redundant parts; this reduces the cost of tools for manufacturing and tests, spare parts etc. In general, the coordination of CIM component systems, especially in the field of CAx- and EDB-systems, can result in a lot of rationalisation.

\section{DISCUSSION}

Any evaluation of the coordination concept in general, and of the data- and application-oriented approaches in particular, has to be based on the requirements for CIM systems. The basic requirement is the capability to connect systems in a heterogeneous hardware and operating system environment; it is important that product data can reside in the component system in which it is created. This stems from a need for the component systems to be able to exploit optimisations in accessing their specialised kind of data. The independence of local tasks and the buffering of global requests are additional desirable requirements. Last, but not least, the incorporation of CAD systems to ensure consistency of geometric and non-geometric data is one of the major problems to be dealt with and has to be examined in detail.

One of the general advantages of coordination approaches, in contrast to integration based approaches, is the resulting autonomy of component systems and the elimination of the necessity to build an integration database and the consequent reduction of the integration effort. Such an integration database would often result in an enterprisewide model which may be too expensive to produce and too difficult to attain. The amount of data redundancy is minimised in the coordination approach and, further, the availability of component systems is not dependent on the availability of a central database.

As discussed in section 5, the application-oriented approach based on operational dependencies is simple to realise and, additionally, facilitates the extension of component system functionality and the inclusion of global applications. Furthermore, there is no need to create global object identifications for new objects of global importance. For a migration of component systems to other hosts, only minimal updates of the system identification in the coordinator's database is necessary and so maximum flexibility can be achieved. To standardise the global object method interface in order to transfer parameters, an exchange format such as STEP can be used. However the specific architecture presented does not support transaction management to ensure the consistency and durability of updates in the event of system or communication failures. To cater for this, the agents would require additional database functionality in line with those of the data-oriented approach presented in section 4.

There are certain forms of inter-system dependencies that can be represented in the data-oriented approach but not in the application-oriented approach. For example, many-to-many relationships between objects of different component systems can be represented by the data dependencies of the Coordination View as described in section 4 ; but there is no way to represent these through method mappings. Further, its use of default reactions for violations of global data consistency mean that the system is more amenable to ad-hoc forms of coordination. On the negative side, the data-oriented approach is less direct in its coordination of user tasks and is less well-suited to 
providing specific extensions of component system functionality. Therefore, it is suggested that the data-oriented approach may be most appropriate in the case of PPS-EDB coordination while the operational one is preferred for functionality extension of CAx and EDB systems (compare figure 4).

For both coordination approaches, the following check list could be used to judge the ease with which a component system could be incorporated into a CIM system. Ideally, a component system should have

- accessibility of the component system's data at the logical level on which this access can be performed, e.g. whether a CAD Agent 'sees' SQL statements or operations on CAD objects;

- the possibility of a control transfer in the case of operations on globally important objects;

- freedom from side effects for all accessible object methods;

- the possibility to use a commonly used communication protocol (e.g. TCP/IP and sockets/RPC);

- the ability to transform a component system's data into a form suitable for network transfer.

\section{CONCLUSIONS}

We have presented two coordination approaches for CIM systems which support cooperative working through the coordination of component system activities as opposed to the traditional approaches based on the integration of data and/or applications. The advantages of a coordination approach stem from the resulting looser coupling of component systems thereby yielding maximal local autonomy and flexibility. An important factor is to support the evolution of CIM systems in terms of the addition and/or replacement of component systems. This is achieved by basing coordination on inter-system dependencies which are stored in a central repository using a global representation language. These dependencies can then be queried and updated directly.

The two approaches described, with their respective prototypes, illustrate two extreme orientations arising from whether inter-system dependencies are based on data or operations. Of course, there is a broad spectrum between these two extremes in which aspects of the two orientations can be combined. We are continuing to investigate these two approaches and their relative merits and consider how best to apply them and combine them in future CIM systems.

\section{ACKNOWLEDGEMENTS}

The work described in this paper is part of the project "Integration of Databases and CIM subsystems" (CIM/Z) funded by KWF (Swiss Federal Commission for the Advancement of Scientific Research). We thank our partners on this project for their contributions to the project as a whole and the CIM/Z system in particular; they are ABB Informatik AG and Sulzer Informatik AG.

\section{REFERENCES}

Beeri, C., Bernstein, P. \& Goodman, N. (1989), 'A Model for Concurrency in Nested Transaction Systems', Journal of the ACM.

Bloomer, J. (1992), Power Programming with RPC, O'Reilly \& Associates, Inc., Sebastopol, CA.

Breitbart, Y., Garcia-Molina, H. \& Silberschatz, A. (1992), 'Overview of Multidatabase Transaction Management', VLDB Journal. 
Brosda, V. (1992), Data Integration of Heterogenous Applications - A Technique for CIM System Implementation, Technical report, IBM Germany, Heidelberg Scientific Center.

Brosda, V. \& Herbst, A. (1992), Die Integrationsdatenbank - Ein Ansatz zur Datenintegration im CIM-Umfeld, Technical report, IBM Germany, Heidelberg Scientific Center, Tiergartenstr. 15, D-69121 Heidelberg.

CAT (1992), CATIA Base - Geometry Interface Reference Manual, SH50-0091-04.

Eversheim, W. \& Grosse-Wienker, R. (1991), Document Management Architecture - A Concept for Integration of Distributed Application Systems in Manufacturing and Engineering, in 'Proc. of the 3rd International Symposium on Systems Research, Informatics and Cybernetics, Baden-Baden, Germany'.

GII (1988), CATIA - Graphics Interactive Interface (GII) Reference Manual, SH50-0020-0.

Gorys, L. T. \& Brauch, A. (1993), TCP/IP Arbeitsbuch: Kommunikationsprotokolle zur Datenübertragung in heterogenen Systemen, 3rd edn, Hüthig Verlag, Heidelberg.

IGE (1988), Initial Graphics Exchange Specification (IGES). Version 4.0.

Jablonski, S., Ruf, T. \& Wedekind, H. (1988), Implementation of a Distributed Data Management System for Manufacturing Applications, in 'Proc. of the IEEE Int. Conf. on Computer Integrated Manufacturing (CIM)', pp. 1928.

Jablonski, S., Ruf, T. \& Wedekind, H. (1990), Concepts and Methods for the Optimization of Distributed Data Processing, in 'Proc. of the IEEE Second Int. Symposium on Databases in Parallel and Distributed Systems, Dublin', pp. 171-180.

Lorie, R. \& Bever, M. (1987), Database Support for Computer Integrated Manufacturing, Technical report, IBM Research Division, San Jose.

Mark, L., Roussopoulos, N. \& Cochrane, R. (1994), Update Dependencies in the Relational Model, submitted for publication.

Montau, R. (1993), 'Integritätsgewinn für die Sachnummerung anhand semantischer Datenmodellierung', Konstruktion 45(10), 321-328.

Montau, R. (1994), 'Sachnummernabbildung: Zugriffsmechanismen und Integritätskontrolle für das Engineering Daten Management', CIM-Management 10(6), 40-44.

Norrie, M. C., Schaad, W., Schek, H.-J. \& Wunderli, M. (1994a), CIM through Database Coordination, in 'Proc. of the Int. Conf. on Data and Knowledge Systems for Manufacturing and Engineering, Hongkong'.

Norrie, M. C., Schaad, W., Schek, H.-J. \& Wunderli, M. (1994b), Exploiting Multidatabase Technology for CIM, Technical Report 219, ETH Zurich.

Norrie, M. C. \& Wunderli, M. (1994), Coordination System Modelling, in P. Loucopoulos, ed., 'Proc. of the 13th Int. Conf. on the Entity Relationship Approach, Manchester, UK', Springer, pp. 474-490.

Par (1993), Pro/ENGINEER User Manuals.

Schaad, W., Montau, R., Wunderli, M., Leonhardt, U. \& Lüthi, A. (1993), Integration von Datenbanken und CIM Subsystemen, Technical report, ETH Zürich. Zwischenbericht KWF Projekt 2308.2.

Schaad, W. \& Schek, H.-J. (1993), Federated Transaction Management Using Open Nested Transactions, in 'Proc. of the DBTA-Workshop on Interoperability of Database Systems and Database Applications, Fribourg, Switzerland'.

Scheer, A.-W. (1990), Computer Integrated Manufacturing - Der computergesteuerte Industriebetrieb, 4th edn, Springer, Berlin.

Schek, H.-J., Weikum, G. \& Schaad, W. (1991), A Multi-Level Transaction Approach to Federated DBS Transaction Management, in 'Proc. of the 1th Int. Workshop of Interoperability in Multidatbase Systems, Kyoto, Japan'.

SET (1984), Standard d'Échange et de Transfert. Revision 1.1.

SET (1985), Automatisation Industrielle Représentation externe des donneés de definition de produits: Specification du Standard d'Échange et Transfert. Version 85-08.Z68-300.

Shooman, M. L. (1983), Software Engineering: design, reliability and management, Computer Science, Mac GrawHill, New York. 
STE (1992), ISO DIS 10303, Product Data Representation and Exchange.

VDA (1987a), Festlegung einer Untermenge von IGES Version 3.0 (VDA-IS). VDMA/VDA 66319.

VDA (1987b), VDA Flächenschnittstelle (VDA-FS). Version 2.0.

Weikum, G. (1991), 'Principles and Realization Strategies of Multilevel Transaction Management', ACM TODS.

Weikum, G. \& Schek, H.-J. (1992), Concepts and Applications of Multilevel Transactions and Open Nested Transactions, in A. Elmagarmid, ed., 'Database Transaction Models for Advanced Applications', Morgan Kaufmann, San Mateo, CA, chapter 13, pp. 515-553.

Wintraecken, J. (1989), The NIAM Information Analysis Method: Theory and Practice, Kluwer Academic Publishers.

\section{BIOGRAPHIES}

Moira Norrie received a B.Sc. in Mathematics from the University of Dundee, an M.Sc. in Computer Science from Heriot-Watt University and a Ph.D. from the University of Glasgow. She is currently a Senior Research Associate in the Database Research Group at the Swiss Federal Institute of Technology (ETH) Zurich. Previously, she has held research and lecturing positions at a number of institutions including the Universities of Edinburgh, Glasgow and Stockholm. Her main research interests are in the area of object data models and semantic interoperability.

Martin Wunderli holds a Master's Degree in Computer Science from the Swiss Federal Institute of Technology (ETH) Zurich and is now a Ph.D. student at ETH. His main interests are foundations of data models, especially object data models. In the CIM/Z project, he has been working on modelling issues since June 1992.

Robert Montau studied mechanical engineering at the University of Karlsruhe. Since 1990, he has been a research assistant at the Institute for Construction and Design Methods of the Swiss Federal Institute of Technology (ETH) Zurich (head: Prof. Dr.-Ing. M. Flemming). His major areas of interest are product data management, CAxtechnologies and computer integrated design models.

Uwe Leonhardt studied computer science at the University of Karlsruhe and completed his studies with a Diploma Work at the Swiss Federal Institute of Technology (ETH) Zurich. Since 1993, he has been working as research assistant at the Institute for Construction and Design Methods of ETH (head: Prof. Dr.-Ing. M. Flemming). His major areas of interest are product data management, CAx-technologies and computer communication.

Werner Schaad is a Ph.D. student at the Swiss Federal Institute of Technology (ETH) Zurich. His research interests include transaction theory in database systems and distributed and federated database systems. He has built a prototype system of a federated database system using the concept of multi-level transactions. In the CIM/Z project, he has been working on transaction issues since 1991 .

Hans-Jörg Schek received an M.Sc. in Mathematics and a Ph.D. in Civil Engineering from the University of Stuttgart. He is currently a Professor of Computer Science at the Swiss Federal Institute of Technology (ETH) Zurich where he is the head of the Database Research Group. Between 1972 and 1983, he was with the IBM Heidelberg Scientific Centre. From 1983 to 1988, he was a Professor of Computer Science at the Technical University of Darmstadt. His main research interests are transaction management and advanced storage services. 